\title{
HLA-A1, A2, B35-Restricted Survivin Peptides/Montanide ISA-51 Vaccine
}

National Cancer Institute

\section{Source}

National Cancer Institute. HLA-A1, A2, B35-Restricted Survivin Peptides/Montanide ISA-

51 Vaccine. NCI Thesaurus. Code C48393.

A peptide vaccine comprised of synthetic HLA-A1, -A2 and -B35 restricted survivin epitopes combined with the adjuvant Montanide ISA-51 with potential antineoplastic activity. Upon administration, HLA-A1, A2, B35-restricted survivin peptides/Montanide ISA-51 vaccine may stimulate a cytotoxic $T$ cell response ag ainst tumor cells that overexpress survivin, resulting in tumor cell lysis. Montanide ISA-51, also known as incomplete Freund's adjuvant or IFA, is a stabilized water-in-oil emulsion adjuvant containing mineral oil with mannide oleate added as a surfactant that non-specifically stimulates cell-mediated immune responses to antigens. 